\title{
Mutations in an Essential U2 Small Nuclear RNA Structure Cause Cold-Sensitive U2 Small Nuclear Ribonucleoprotein Function by Favoring Competing Alternative U2 RNA Structures
}

\author{
MARY I. ZAVANELLI, JESSICA S. BRITTON, A. HALLER IGEL, AND MANUEL ARES, JR.* \\ Biology Department, Sinsheimer Laboratories, University of California, Santa Cruz, Santa Cruz, California 95064 \\ Received 1 October 1993/Returned for modification 30 November 1993/Accepted 13 December 1993
}

\begin{abstract}
Mutations in stem-loop IIa of yeast U2 RNA cause cold-sensitive growth and cold-sensitive U2 small nuclear ribonucleoprotein function in vitro. Cold-sensitive $\mathrm{U} 2$ small nuclear RNA adopts an alternative conformation that occludes the loop and disrupts the stem but does so at both restrictive and permissive temperatures. To determine whether alternative U2 RNA structure causes the defects, we tested second-site mutations in U2 predicted to disrupt the alternative conformation. We find that such mutations efficiently suppress the cold-sensitive phenotypes and partially restore correct U2 RNA folding. A genetic search for additional suppressors of cold sensitivity revealed two unexpected mutations in the base of an adjacent stem-loop. Direct probing of RNA structure in vivo indicates that the suppressors of cold sensitivity act to improve the stability of the essential stem relative to competing alternative structures by disrupting the alternative structures. We suggest that many of the numerous cold-sensitive mutations in a variety of RNAs and RNA-binding proteins could be a result of changes in the stability of a functional RNA conformation relative to a competing structure. The presence of an evolutionarily conserved $U 2$ sequence positioned to form an alternative structure argues that this region of $\mathrm{U} 2$ is dynamic during the assembly or function of the $\mathrm{U} 2$ small nuclear ribonucleoprotein.
\end{abstract}

RNA-RNA interactions between small nuclear RNAs (snRNAs) or between snRNAs and the pre-mRNA play critical roles in the accuracy and efficiency of splicing (for reviews, see references 15 and 16). Not all of these interactions are established simultaneously, nor do they persist once established. Rather, interactions are formed, modified, disrupted, and replaced during spliceosome assembly and splicing. The dynamic relationship between $\mathrm{U} 4$ and $\mathrm{U} 6$ has been known for some time $(15,16)$, and more recently it has been shown that U2 also interacts with U6 $(11,26,47)$. U6 may interact simultaneously with $U 4$ and $U 2(15,16,46)$, but part of the proposed interaction between $\mathrm{U} 4$ and $\mathrm{U} 6$ must be disrupted if U6 is to interact with $\mathrm{U} 2(26,27)$, suggesting that multiple RNA structural rearrangements are important for progression of the spliceosome through the process of splicing.

The presence of dynamic RNA-RNA interactions within a ribonucleoprotein (RNP) complex like the spliceosome suggests that the snRNAs themselves may need to adopt more than one RNA conformation in order to execute their functions during splicing. Given the severe constraint of satisfying multiple partners, a high degree of sequence conservation is expected of dynamic RNA, making phylogenetic comparisons of limited use in uncovering dynamic RNA segments. Where variation is tolerated, dynamic RNA segments will be conserved in a fashion consistent with multiple secondary structures. An example of this latter pattern occurs in the region of U2 snRNA downstream of the site of intron branchpoint interaction. Phylogenetic variation in this region of U2 supports three different secondary structures: a pseudoknot and two different conformations lacking a pseudoknot $(1,2,18,22)$.

\footnotetext{
* Corresponding author. Mailing address: Sinsheimer Laboratories, University of California, Santa Cruz, CA 95064. Phone: (408) 4594917. Fax: (408) 459-3737. Electronic mail address: ares( $a$ orchid. ucsc.edu.
}

Structure-function studies in this region of $\mathrm{U} 2$ show that only one of the structures, that containing stem-loop IIa, is absolutely essential for growth, arguing that the potential to form the others must be conserved for an accessory or overspecified function (2). Direct probing of yeast U2 structure in vivo confirms that the bulk of $\mathrm{U} 2$ in the cell adopts the essential structure (2); however, the alternative structure can form under certain circumstances, suggesting that this region of $\mathrm{U} 2$ snRNA is dynamic (49).

An unanticipated phenotype associated with single base changes that destabilize the essential structure is cold-sensitive growth (2). Cold sensitivity is also observed in cell splicing extracts, allowing the demonstration of a role for stem IIa in the critical step of assembly of U2 snRNPs into the spliceosome in vitro (49). Structure probing experiments show that the bulk of U2 snRNA is misfolded in the cold-sensitive mutants, so that the RNA adopts the other phylogenetically conserved structure. Surprisingly, the misfolded form predominates at both permissive and restrictive temperatures, suggesting that interconversion between the two folded forms must be rate limiting for growth at restrictive temperatures (49). The phenotype of cold sensitivity has been uncovered on demand in genetic studies of RNA and RNA-binding protein function $(10,17,21,35,37,42,44)$ and also in a surprising number of cases in which it was unanticipated $(2,9,13,25,33,34,40,45$, 48). In some of these cases, cold sensitivity is caused by the loss of a gene product that influences RNA structure $(13,37,45$, 48 ), ruling out differential function of a mutant gene product at high temperatures as a cause of the cold sensitivity. Coldsensitive mutations are not restricted to RNA-based systems, but it seems possible that the disruption of RNA structure, either by direct mutation or by the alteration of activities that modulate RNA structure, can lead to cold sensitivity by a mechanism that would have no corollary in protein structure.

To understand the relationship between alternative U2 
snRNA foldings and the cold-sensitive phenotype, we have constructed $\mathrm{U} 2$ alleles containing second mutations predicted to prevent the alternative folding and tested them for the ability to suppress cold sensitivity. We have found that the conserved complementarity is required for the manifestation of cold-sensitive growth. Certain severe disruptions of stem IIa could be suppressed by mutations in the conserved complementarity, but strains lacking any ability to form stem IIa proved inviable even though the nucleotides of loop IIa were accessible, demonstrating a requirement for the stem. We conducted a genetic screen to isolate additional intragenic suppressors and found that the effects of all of the suppressors can be attributed to their enhancement of the ability of the RNA to adopt a conformation containing stem IIa. The results demonstrate that alternative RNA folding can cause coldsensitive function of RNA and that in the case of U2 snRNA, for which the potential to form the alternative structure is conserved, disrupting the alternative folding relieves the coldsensitive defect. This finding suggests that alternative RNA folding may provide a general explanation for the common occurrence of cold-sensitive mutations in RNA and RNAbinding proteins.

\section{MATERIALS AND METHODS}

Yeast strains. Saccharomyces cerevisiae strains were grown according to standard procedures (41). Yeast strain ALYSGA is MATa leu2-3,112 ura3-52 his4-619 snr20::URA3 lys2 and carries plasmid YCpLYS2-U2G53A. Strain BLYSGA carries the same markers as ALYSGA except that it is MAT $\alpha$. ALEUGA and BLEUGA are derivatives of ALYSGA and BLYSGA created by transformation with YCpLEU2-U2G53A and removal of plasmid YCpLYS2-U2G53A by shuffling on medium containing $\alpha$-aminoadipate (Aldrich) as the primary nitrogen source (6). These strains were used in the screen for second-site suppressor mutations. Strain HI70 is MATa leu23,112 ura3-52 his4-619 snr20::URA3 lys2 carrying YCpLYS2U2C121U (2). HI70 derivatives used for growth phenotypes and structure-probing experiments were created by transformation of a centromeric $L E U 2$ plasmid carrying the $\mathrm{U} 2$ allele of interest and shuffling on $\alpha$-aminoadipate. Strain MA90 is MATa leu2-3,112 ura3-52 his4-619 lys2 snr20-dl-BD (mini-U2 [19]). MA90 derivatives used for structure probing of lethal U2 alleles were created by transformation of a centromeric LEU2 plasmid carrying the desired $\mathrm{U} 2$ allele and selection for leucine independence. Splicing extracts were prepared from strain BJ81, which is MATa GAL2 leu2-3,112 ura3-52 trp1 pep4-3 prb1 prc1 and contains a glucose-repressible wild-type U2 allele integrated into the chromosome (29). When transformed with a $\mathrm{U} 2$ allele on a centromeric $L E U 2$ plasmid (in this case, wild-type, C62U, and C62U G100A) and grown on glucose, only the plasmid-borne $\mathrm{U} 2$ gene is expressed.

Oligonucleotide-directed mutagenesis. Oligonucleotide-directed mutagenesis was performed as described by Kunkel (23). Secondary mutations were introduced into U2 by sitedirected mutagenesis of previously constructed M13 clones (2) containing the desired primary mutation. The oligonucleotides used to introduce the secondary mutations were tmB' (CAAAAAATGTGTATTGTAGTTAATTAAAAG) and G100A (GTATTGTAATAAATTAAAAGG).

In vivo structure probing. Dimethyl sulfate (DMS) modification and RNA analysis were carried out as previously described $(2,49)$. Yeast cultures were grown to an optical density at $600 \mathrm{~nm}$ of 0.8 to 2.0 in YEPD or SCD medium. Cells were treated with DMS for $4 \mathrm{~min}$ at $30^{\circ} \mathrm{C}$, and then the modification reactions were stopped by the addition of $0.6 \mathrm{M}$ $\beta$-mercaptoethanol. Total RNA was extracted as described below, and the modified nucleotides were mapped by primer extension from an end-labeled oligonucleotide, YSTR, which is complementary to U2. To monitor the structure of lethal U2 alleles in vivo, strain MA90 was used. MA90 carries a mini-U2 (19) in the chromosome as its only functional allele. This strain was transformed with $L E U 2$ plasmids bearing the $\mathrm{U} 2$ alleles of interest and maintained by growth in SCD lacking leucine. Yeast cultures were grown and treated as described above, and primer extension from end-labeled YSTR was used to map the modified mutant U2s. The mini-U2 lacks the YSTR priming site and therefore does not interfere with the structural analysis.

RNA extraction and analysis. Total RNA was extracted by a hot phenol method as described previously $(2,49)$. Cell pellets were resuspended in $0.5 \mathrm{ml}$ of AK buffer (AK buffer is $1 \mathrm{~g}$ of triisopropylnaphthalene sulfonic acid, $6 \mathrm{~g}$ of sodium $p$-aminosalicylate, $1.17 \mathrm{~g}$ of sodium chloride, and $6 \mathrm{ml}$ of phenol per $100 \mathrm{ml}$ of buffer $)$. Hot $\left(65^{\circ} \mathrm{C}\right)$ phenol $(0.5 \mathrm{ml})$ was added, and the mixture was vortexed for $30 \mathrm{~s}$ and incubated at $65^{\circ} \mathrm{C}$ for 30 min, with two additional 30 -s vortexes at 10 -min intervals. The mixture was incubated on ice for $5 \mathrm{~min}$ and then spun for $5 \mathrm{~min}$ in a microcentrifuge. The aqueous phase was removed and extracted once with phenol-chloroform-isoamyl alcohol (25: 24:1) and once with chloroform. Sodium acetate ( $\mathrm{pH}$ 5.2) was added to a final concentration of $0.3 \mathrm{M}$, and the RNA was precipitated by the addition of 3 volumes of $95 \%$ ethanol. The pellet was resuspended in water, and the nucleic acid concentration was estimated by determining the $A_{260}$ and $A_{280}$.

Primer extensions were performed as described previously (2), using the end-labeled oligonucleotide YSTR (ATTATTT TGGGTGCCAA; annealing temperature, $37^{\circ} \mathrm{C}$ ). Oligonucleotide primers were labeled at their $5^{\prime}$ ends with $\left[{ }^{32} \mathrm{P}\right]$ phosphate, using $\left[\gamma^{32} \mathrm{P}\right] \mathrm{rATP}$ and polynucleotide kinase.

Splicing complex analysis. Yeast whole-cell extracts were prepared essentially as described by Lin et al. (24) from strain BJ81 derivatives grown in YEPD at $30^{\circ} \mathrm{C}$ to an $A_{600}$ of 3.0 to 5.0. Wild-type yeast actin pre-mRNA substrate was synthesized in vitro, using T7 RNA polymerase as described by Milligan and Uhlenbeck (28) with use of a previously constructed T7 actin clone (49). The transcript was gel purified and used at a specific activity of 500 to $2,000 \mathrm{cpm} / \mathrm{fmol}$.

Splicing complexes were prepared with synthetic radiolabeled yeast actin pre-mRNA and analyzed on nondenaturing polyacrylamide gels as previously described (49). Reactions were assembled at $0^{\circ} \mathrm{C}$, and aliquots were removed at $0,2,5$, or $10 \mathrm{~min}$ after shifting to either 12,15 , or $18^{\circ} \mathrm{C}$.

Isolation of suppressors. Strains ALYSGA and BLYSGA were mutagenized with ethyl methanesulfonate (Sigma) to $90 \%$ lethality by standard procedures (3) and plated at $18^{\circ} \mathrm{C}$. Cold-resistant clones were selected and mated with the $L E U^{+}$ lys $^{-}$strain of the opposite mating type (BLEUGA and ALEUGA, respectively). Diploids containing both plasmids were obtained by selection at $30^{\circ} \mathrm{C}$ on synthetic complete medium (SCD) lacking lysine and leucine and were streaked onto medium containing $\alpha$-aminoadipate as the primary nitrogen source (6). Strains that lost the LYS2 plasmid were then streaked on YEPD medium and grown at $18^{\circ} \mathrm{C}$ to test for loss of suppression. Those strains which retain cold resistance after loss of the mutagenized plasmid are presumed to have a dominant suppressor mutation in the chromosome. Those strains which regain cold sensitivity after loss of the LYS2 plasmid carry a plasmid-borne reversion mutation presumed to be within the U2 gene.

Characterization of intragenic suppressors. To distinguish true revertants (A53G) from second-site intragenic suppres- 
sors, we used primer extension of total RNA with end-labeled oligonucleotide YSTR complementary to U2 (see above). ddC was included in these reactions to generate stops at $\mathrm{G}$ residues in the $5^{\prime} 110$ nucleotides (nt) of U2. Strains that did not contain a $\mathrm{G}$ at position 53 were then sequenced by using reverse transcription reactions containing each of the four dideoxynucleotides to identify mutations which might confer the suppressor phenotype. The 950-bp HindIII fragment of each suppressor allele was then subcloned into YCp U2-3' (19) and completely sequenced from positions -329 to +624 to ensure that no additional mutations had been introduced by the mutagenesis. The phenotypes of the subcloned suppressed alleles were retested by transformation into strain HI70 and plasmid shuffling. The shuffled strains were then streaked on YEPD medium and tested for growth phenotypes at various temperatures.

\section{RESULTS}

Mutations in a region complementary to loop IIa suppress cold-sensitive growth. We have previously shown that a U2 RNA misfolding phenotype is associated with cold-sensitive mutations of U2 stem IIa (49). The destabilization of stem IIa allows the stable formation of an alternative RNA structure involving pairing between the loop IIa (nt 54 to 61) and a phylogenetically conserved sequence (nt 98 to 105) complementary to the loop (Fig. 1A). To determine whether the direct cause of cold sensitivity is the alternative folding of the RNA or the mismatched base pair in stem IIa, we constructed and tested second-site mutations that disrupt the conserved complementarity for the ability to suppress cold sensitivity of stem IIa mutations. We reasoned that if such second mutations suppressed cold sensitivity in the presence of a stem IIa mismatch, then the mismatch itself could not be the direct cause of the cold sensitivity.

The locations of these mutations in the secondary structure model for yeast U2 snRNA are shown in Fig. 1A. Growth at $18^{\circ} \mathrm{C}$ of cells carrying a $\mathrm{U} 2$ gene with a single point mutation of either G-53 to A (G53A) or C62U in stem IIa is impaired, whereas the compensatory double mutant G53A C62U grows like the wild type (2) (Fig. 1B). Mutation of G100A is able to suppress the cold sensitivity of both the G53A and C62U mutations, as demonstrated by the wild-type growth of the double mutants G53A G100A and C62U G100A observed at $18^{\circ} \mathrm{C}$ (Fig. 1B). A triple mutation in the conserved complementarity (tmB'; Fig. 1A) is also able to suppress the cold-sensitive phenotype (Table 1). This result provides genetic evidence that perturbation of stem IIa in the primary mutants by the A-C or G-U mismatch does not by itself cause the low-temperature growth defect.

Cold sensitivity of splicing complex formation is suppressed in vitro. The $\mathrm{C} 62 \mathrm{U}$ and $\mathrm{G} 53 \mathrm{~A}$ mutations render the stable association of U2 snRNPs with pre-mRNA cold sensitive in vitro (49). To determine whether the G100A mutation acts to restore the ability of C62U mutant snRNP to associate stably with pre-mRNA during splicing complex assembly, we prepared splicing extracts from the C62U G100A double mutant and monitored splicing complex formation at different temperatures (Fig. 2). Splicing complexes that contain the U2 snRNP have been described by others $(7,31,32,38,39)$ and are labeled 1 and 2 in Fig. 2. As demonstrated previously (49), the rate of stable addition of the U2 snRNP to pre-mRNA in the cold is reduced by the $\mathrm{C} 62 \mathrm{U}$ mutation relative to the wild type (Fig. 2). Extracts prepared from the double mutant C62U G100A show wild-type levels of U2-containing complexes,

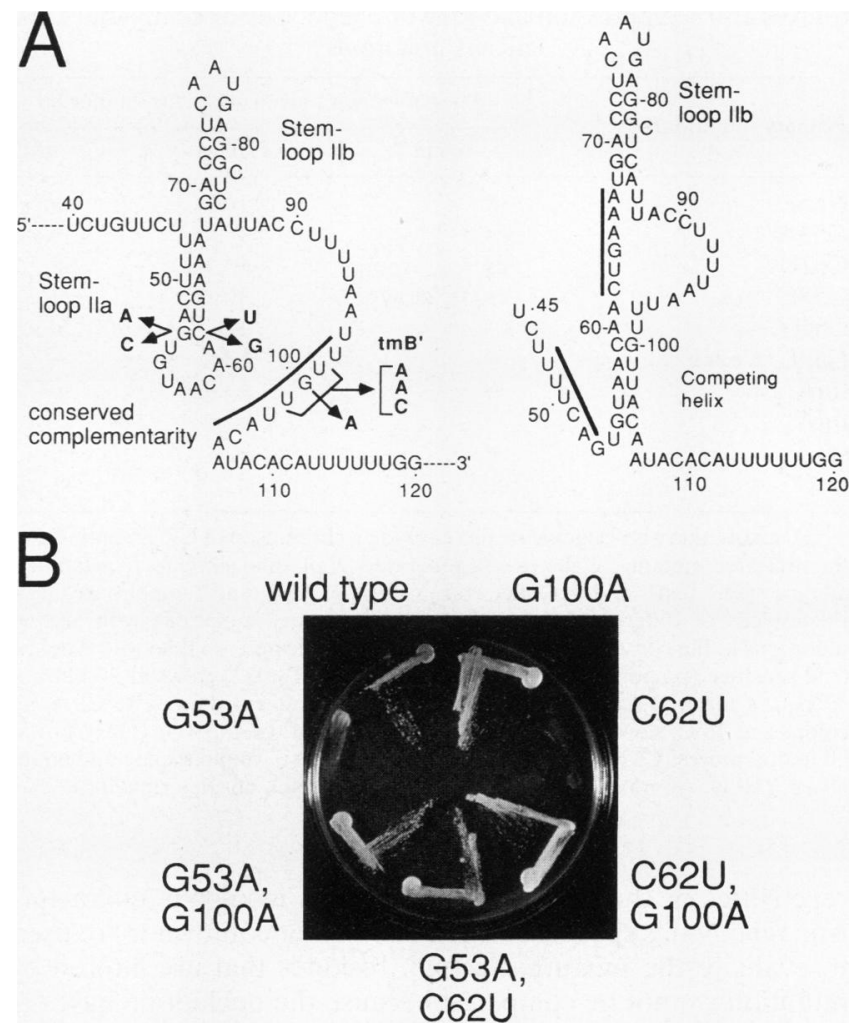

FIG. 1. Mutations in the conserved complementarity suppress cold sensitivity of stem IIa mutants. (A) Secondary structure models of yeast U2 snRNA and sequence changes in the mutants. The sequence of yeast U2 stRNA from positions 40 through 120 is shown folded into the secondary structure model of Ares and Igel (2) on the left and in the alternative structure of Keller and Noon (22) on the right. Stem-loop IIa (nt 48 to 67), stem loop IIb (nt 68 to 85), and the conserved complementarity (nt 98 to 105 , heavy line) are indicated. Sequence changes generated by site-directed mutagenesis are indicated. Mutation of the 53-62 base pair enhances formation of the alternative structure at all temperatures (49). (B) Growth phenotype at $18^{\circ} \mathrm{C}$ of yeast strains carrying U2 mutations. An otherwise isogenic set of strains carrying a $\mathrm{U} 2$ gene disruption in the chromosome and the indicated $\mathrm{U} 2$ allele on a centromere plasmid was constructed by plasmid shuffling. Strains were streaked on YEPD medium and incubated for 4 days at $18^{\circ} \mathrm{C}$.

demonstrating that suppression by G100A occurs at the level of stable U2 snRNP binding to the assembling spliceosome.

Second-site mutations suppress alternative folding of mutant U2 snRNAs in vivo. To determine the impact of the G100A and tmB' suppressor mutations on the folded structure of U2 snRNA, we monitored nucleotide accessibility by using a chemical probe $(20,30)$. This approach relies on the methylation of unpaired $\mathrm{A}$ and $\mathrm{C}$ residues by DMS and can be applied in vivo because of the ability of DMS to penetrate and react with RNA in yeast cells $(2,49)$. Modified residues in RNA are subsequently mapped as strong stops to reverse transcription $(20,30)$. In evaluating these experiments, it is important to keep several things in mind. First, the data represent a superimposition of the reactivities of all individual conformations present during the time of modification. In samples that contain a single predominant conformation, the patterns are readily interpretable, but when the population is composed of two equally represented conformers, the data become difficult to read. In cases for which the pattern of 
TABLE 1. Suppression and growth phenotypes of compound U2 snRNA mutations ${ }^{a}$

\begin{tabular}{|c|c|c|c|}
\hline \multirow{2}{*}{ Primary mutation(s) } & \multicolumn{3}{|c|}{ Phenotype with given secondary mutation } \\
\hline & None & G100A & $\operatorname{tmB}^{\prime}$ \\
\hline None & + & + & + \\
\hline G53A & cs, ts* & + & + \\
\hline C62U & cs & + & + \\
\hline G53C & cs, ts, slow & + & - \\
\hline C62G & - & + & Slow \\
\hline G53C, C62G & + & ND & - \\
\hline $\operatorname{tm} A$ & - & + & + \\
\hline $\operatorname{tm} \mathrm{A}^{\prime}$ & - & ts & $(+)$ \\
\hline smA & + & + & \\
\hline $\operatorname{tm} C^{\prime}$ & sl. cs & sl. cs & sl. cs \\
\hline
\end{tabular}

a A set of otherwise isogenic strains carrying a chromosomal U2 disruption and the indicated mutant $U 2$ alleles was generated by plasmid shuffling on $\alpha$-aminoadipate (see text) and streaked on YEPD at different temperatures (2). Phenotypes of different combinations of 10 primary mutations with second mutations in the conserved complementarity are indicated. + , Like wild type; cs, cold sensitive (pinpoint colonies after 4 days at $18^{\circ} \mathrm{C}$ ); ts*, grows at 36 but not $37^{\circ} \mathrm{C}$ (see text); s1. cs, small colonies $(<0.5 \mathrm{~mm})$ after 4 days at $18^{\circ} \mathrm{C}$; ts, no colonies at $36^{\circ} \mathrm{C}$; slow, small colonies relative to wild type at $30^{\circ} \mathrm{C}(\mathrm{G} 53 \mathrm{C})$ or at all temperatures $\left(\mathrm{C} 62 \mathrm{G}, \mathrm{tmB}^{\prime}\right) ;(+)$, grows poorly on $\alpha$-aminoadipate and not at all on YEPD; -, no colonies at any temperature; ND, not determined.

reactivities of the individual conformers is known, characteristic reactivities specific to one or the other conformer are used to evaluate the mixture. Second, residues that are altered by mutation cannot be compared because the nucleotide bases $\mathrm{A}$

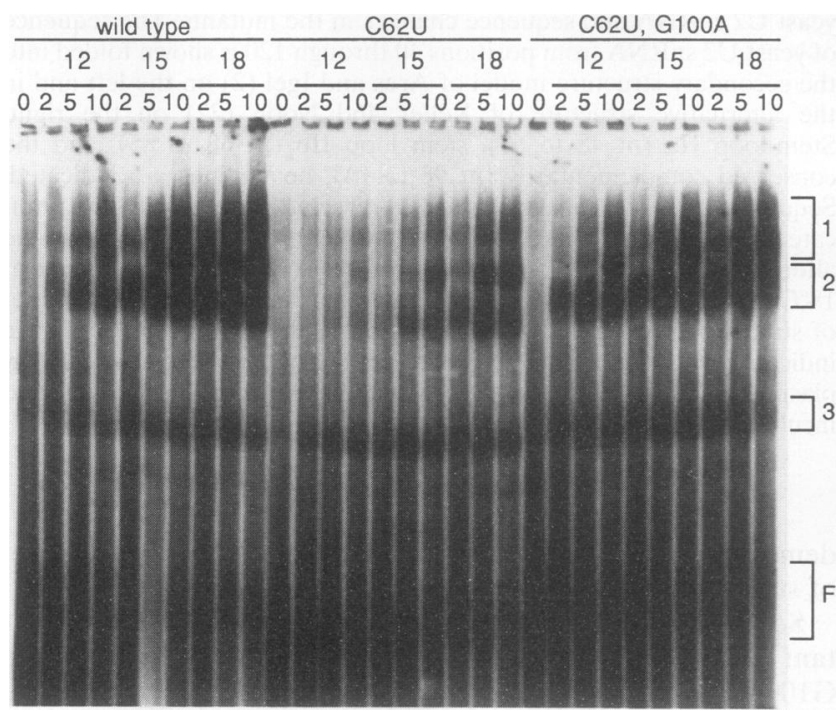

FIG. 2. Time course of splicing complex formation at different temperatures in extracts containing mutant U2 snRNPs. Splicing extracts made from cells expressing only the indicated $\mathrm{U} 2$ allele were incubated with synthetic radiolabeled yeast actin pre-mRNA and analyzed on nondenaturing polyacrylamide-agarose gels as described previously (49). Bracket $F$ indicates migration of free pre-mRNA. Bracket 3 indicates a $\mathrm{U} 2$-independent complex that forms rapidly on ice and in the absence of ATP. Bracket 2 indicates the first U2dependent complex equivalent to the U2 snRNP containing complex III (32) or B (7). Bracket 1 indicates the position of later-assembling complexes that contain reacted substrate as well as $\mathrm{U} 2$ and other snRNPs, equivalent to complexes I and II (32) or the A complexes (7; for details, see reference 49 ). Reactions were assembled at $0^{\circ} \mathrm{C}$, and aliquots were removed at 2,5 , or $10 \mathrm{~min}$ after a shift to either 12,15 , or $18^{\circ} \mathrm{C}$. and $\mathrm{C}$ are reactive, but $\mathrm{G}$ and $\mathrm{U}$ are usually not. This means that occasionally an apparent new reactivity or protection is due to the mutation of $G$ to $A$ (new reactivity) or $C$ to $U$ (new protection). Third, while the loop IIa nucleotides are accessible, the loop is almost certainly structured, as evidenced by the hyperreactivity of A-60 and the partial protection of A-57 and A-58. The loop sequence has a characteristic phylogenetic conservation pattern, but in most organisms, Watson-Crick covariation is not observed. For example, in Saccharomyces cerevisiae, even though Watson-Crick pairing between U-54 and A-61 could extend stem IIa, we draw them as part of the loop, because in other organisms they are U-G, A-U, U-U, $\mathrm{U}-\mathrm{C}$, or A-C $(1,18)$. A-61 is relatively protected in yeast cells and behaves as though it is paired (2). Fourth, the residues near the ends of a helix will tend to be more reactive than those in the middle of the helix, presumably because breathing of the ends renders them more accessible.

Stem-loop IIa is a feature observed in the bulk of wild-type $\mathrm{U} 2$ in vivo $(2,5,49)$ (Fig. 3 ). The G53A or C62U mutation increases the reactivity of adjacent stem IIa residues C-51 and A-52, while A-57, A-58, C-59, and A-60 in loop IIa and A-103 and $\mathrm{C}-104$ in the conserved complementarity become protected (49) (Fig. 3). We interpret this altered pattern of reactivity to mean that the majority of the $\mathrm{U} 2$ is now folded such that stem IIa is disrupted and the loop IIa nucleotides have paired to the downstream conserved complementarity (49). This alternative conformation is similar to that proposed for U2 snRNA by Keller and Noon (22) on the basis of phylogenetic data demonstrating the conservation of complementarity between loop IIa (nt 54 to 61) with the region downstream (nt 98 to 105). We previously showed that the alternative structure predominates in mutant cells grown at 18 , 30 , or $36^{\circ} \mathrm{C}$, suggesting the possibility of low-temperaturedependent kinetic trapping as an explanation for heat-dependent (cold-sensitive) growth (49).

The structure of the loop, as assayed by the reactivities of $\mathrm{nt}$ 57 to 60 , is restored by the G100A mutation in the competing helix, although because of the A-C (in G53A) or G-U (in C62U) mismatch remaining in stem IIa, the protection of stem nucleotides C-51 and A-52 is incomplete (Fig. 3). In addition, A-103 and C-104 regain reactivity (and the new A residue at position 100 is also reactive), consistent with the disruption of the competing helix. Similar results are obtained with the triple mutation tmB' (Fig. 3). We conclude that the ability of the conserved complementarity to form a helix with loop IIa is disrupted by the G100A and tmB' mutations. Stem IIa nucleotides remain more reactive in the suppressed double mutants than in the wild type in part because of the base mismatch caused by the primary mutation.

Disruption of the conserved complementarity suppresses lethal mutations in stem IIa but not a mild cold-sensitive mutation elsewhere in U2 snRNA. To explore the allele specificity of suppression of cold sensitivity by mutations in the conserved complementarity, we tested additional cold-sensitive mutations in $\mathrm{U} 2$ for suppression by the G100A and $\mathrm{tmB}^{\prime}$ mutations (Table 1). One class contains stem IIa point mutations that are severely growth deficient (G53C) and lethal (C62G), while the another class contains a cold-sensitive triple mutation elsewhere in $\mathrm{U} 2$ ( $\left.\mathrm{tmC}^{\prime}\right)$. By itself neither G100A nor tmB' has obvious growth defects, and neither can suppress the cold-sensitive phenotype of the $\operatorname{tmC}^{\prime}$ mutation (Table 1). G100A suppresses the lethal phenotype of the C62G mutation as well as the cold-sensitive, heat-sensitive, and slow-growth effects of the G53C mutation. In the case of tmB', efficient suppression of both the G53A and C62U mutations is observed. Suppression of the lethal C62G by the tmB' mutation 


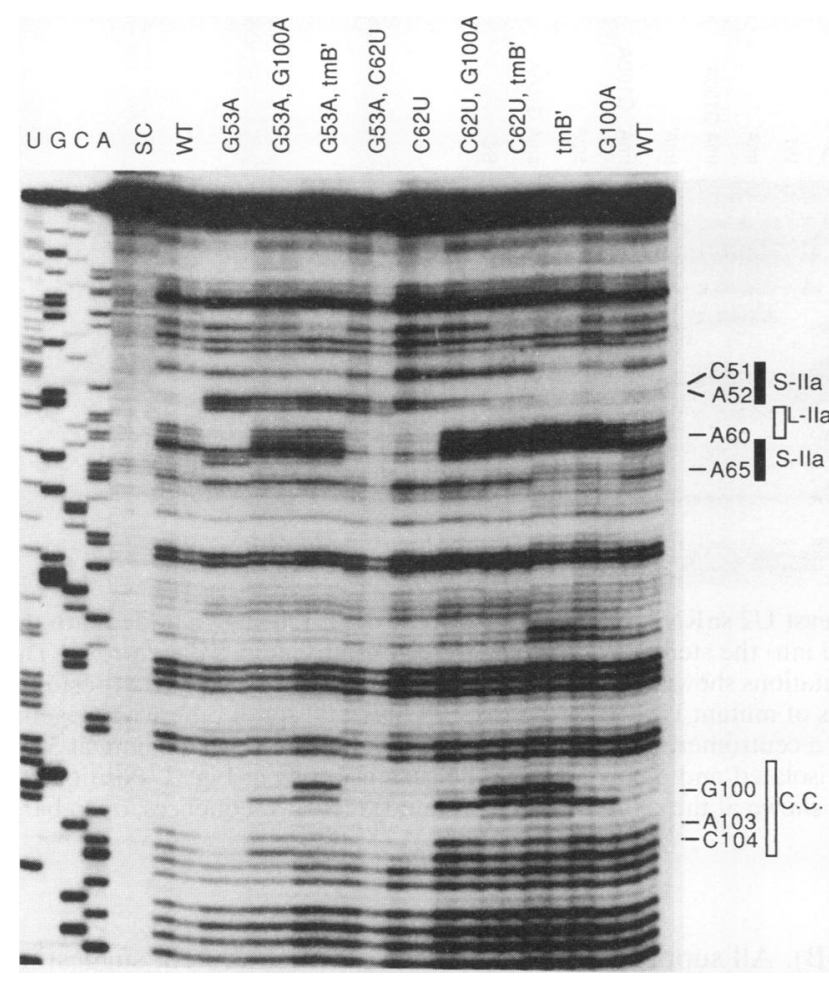

FIG. 3. Methylation protection analysis of mutant U2 snRNA secondary structure in vivo. Cultures of a set of otherwise isogenic yeast strains carrying a $\mathrm{U} 2$ gene disruption in the chromosome and the indicated $\mathrm{U} 2$ allele on a centromere plasmid were grown in liquid YEPD medium at $30^{\circ} \mathrm{C}$ and exposed to DMS, and then the reaction was quenched and RNA was isolated as described previously (49). RNA was analyzed by primer extension using the YSTR oligonucleotide as a primer. Lanes $U, G, C$, and A represent reverse transcription reactions of unmodified wild-type RNA to which dideoxynucleotides were added (ddA, ddC, ddG, and ddT, respectively) to generate a sequence ladder. Lanes SC are stop control reactions in which DMS was added after the quench solution to prove that modification does not occur during the potentially denaturing extraction procedure. Pairs of adjacent lanes are loaded with the same sample to account for occasional variation in loading volume. Note that reverse transcription stops due to methylation are one nucleotide shorter than those due to incorporation of a dideoxynucleotide. Positions of migration of the modification-induced stops for the indicated nucleotides are shown at the right. Black bars, stem IIa (S-IIa) sequences; open bars, loop IIa (L-IIa) and the conserved complementarity (C.C.). WT, wild type.

is only partial but is temperature independent, as the cells grow more slowly than wild-type cells at all temperatures. Combination of G53C with tmB' $^{\prime}$ is lethal, even when the G53C mutation is in combination with the compensatory $\mathrm{C} 62 \mathrm{G}$ mutation (the G53C C62G double mutant is viable and not temperature sensitive), suggesting specific negative interactions between the $\mathrm{tmB}^{\prime}$ and $\mathrm{G} 53 \mathrm{C}$ mutations. From these genetic data, we conclude that the suppression afforded by mutation of the competing helix is general only for mutations that destabilize stem IIa.

Previous data demonstrated that U2 stem-loop IIa is required for U2 function $(2,49)$. The data showing that stem IIa mutants are viable provided that the conserved complementarity to the loop is destroyed (Table 1) suggest that stem IIa may be required only to present the loop nucleotides, that is, to ensure that the loop is accessible. To distinguish between a requirement for stem IIa or a simple requirement for accessi- bility of the loop IIa nucleotides, triple mutations in stem IIa were tested in conjunction with mutations in the conserved complementarity, with the goal of eliminating stem IIa while leaving the loop nucleotides unpaired. If stem IIa is itself essential, these compound mutants should be lethal. If the availability of the loop nucleotides is sufficient for U2 function, then these mutants should prove viable. The G100A and tmB' mutations in the conserved complementarity were introduced into lethal $\mathrm{U} 2$ alleles carrying triple mutations ( $\operatorname{tmA}$ and $\left.\operatorname{tmA} A^{\prime}\right)$ in stem IIa, as well as the viable, compensatory sextuple mutation (smA, made by combining tmA with tmA' ${ }^{\prime}$ [2]) (Table 1; Fig. 4A). The tmA' mutation is not suppressed by either the G100A or tmB' mutation; however, the tmA G100A mutant is viable and heat sensitive. The $\operatorname{tm} A \operatorname{tmB}^{\prime}$ mutant is viable but extremely slowly growing. The finding that a triple mutation in the stem can grow at all may seem at odds with the conclusion that the stem is essential (2), but in fact the tmA mutant retains the potential to form a weak stem IIa involving two G-U base pairs, whereas tmA' retains no apparent ability to form a stem IIa (Table 1; Fig. 4A). These results demonstrate a requirement for stem IIa but do not exclude the possibility that the accessibility of the loop nucleotides is an important feature of this region for $\mathrm{U} 2$ function.

Accessibility of loop IIa nucleotides is not sufficient for U2 function. To validate interpretations made on the basis of genetics, the folded structures of the U2 stem IIa triple mutations, alone and in combination with mutations in the conserved complementarity (G100A and tmB'; Fig. 4A), were probed in vivo (Fig. 4B). Because of the lethal phenotype of many of these mutants, structure probing was conducted in MA90, a yeast strain in which the only functional copy of $U 2$ is a truncated version (mini-U2) that lacks the priming site found in the full-length mutant U2 (19). The mutant U2 genes can be transformed into this strain and maintained by growth in selective media. Total RNA isolated from these strains after treatment with DMS will contain both the mutant and the mini-U2s, but primer extension will occur only from the mutant snRNAs, and thus their folded structure can be probed despite their lethality.

The tmA mutation disrupts U2 stem IIa, as shown by increased accessibility of stem nucleotides (A-50, A-65, A-66, and A-67) to the chemical probe compared with the wild-type RNA (Fig. 4B). Nucleotides in loop IIa and the conserved complementarity become protected from modification (A-57, A-58, and C-59 in loop IIa [Fig. 4B]; G-100, A-103, and C-104 in the conserved complementarity [data not shown]). In the tmA G100A mutant, the protection of loop IIa and downstream nucleotides is abolished, but the modification of the stem nucleotides appears to remain unchanged aside from a slight protection of C-62. The tmA $\mathrm{A}^{\prime}$ mutant alone also shows increased reactivities of the stem IIa nucleotides (C-51, A-52, C-63, and A-64) and protection of the loop and downstream nucleotides as in the tmA mutant (Fig. 4B). The tmA' G100A mutant also demonstrates restored reactivities of the loop and downstream nucleotides, while the stem nucleotides remain accessible (Fig. 4B). The compensatory mutant smA shows wild-type levels of reactivity in stem-loop IIa and the downstream conserved complementarity when tested alone. In conjunction with the G100A mutation, this pattern of reactivity is identical aside from the increased protection observed at nucleotides C-62 and C-63. Because the modification data demonstrate that the loop IIa region is accessible in the lethal tmA' G100A mutant RNA, we conclude that the structural requirements for this region of $\mathrm{U} 2$ consist of more than simply that the loop nucleotides be available; an intact stem IIa must also be present. 


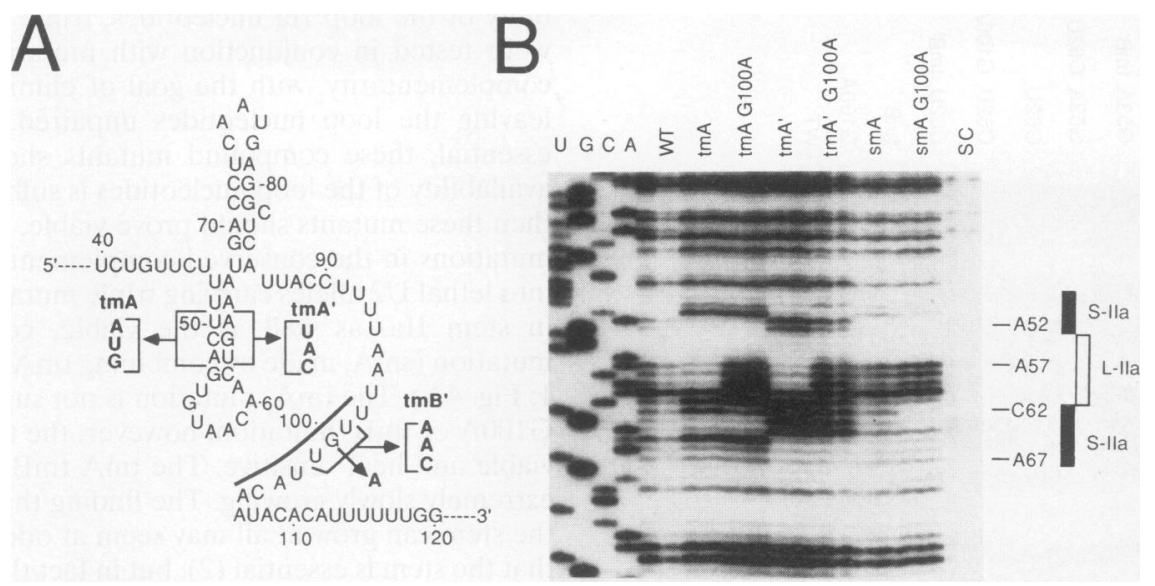

FIG. 4. Triple mutations combined with a suppressor. (A) Sequence of yeast U2 snRNA from positions 40 through 120 shown folded into the secondary structure model of Ares and Igel (2). Triple mutations introduced into the stem IIa and stem IIb regions are indicated by arrows. The compensatory sextuple mutant smA consists of both of the stem IIa triple mutations shown. The conserved complementarity to loop IIa (positions 98 to 105) is indicated by the heavy line. (B) Modification protection analysis of mutant U2 RNAs in vivo. Cultures of a set of otherwise isogenic yeast strains carrying a chromosomal mini-U2 and the indicated $\mathrm{U} 2$ allele on a centromere plasmid were grown in liquid selective medium at $30^{\circ} \mathrm{C}$ and exposed to DMS, and then the reaction was quenched and RNA was isolated and analyzed by primer extension as in Fig. 2. Positions of migration of the modification-induced stops for the indicated nucleotides are shown at the right. Black bars, stem IIa (S-IIa) sequences; open bars, competing helix sequences. L-IIa, loop IIa.

Genetic selection for cold resistance reveals new mutations in loop IIa and stem IIb that act as suppressors. To search for other second-site mutations in $\mathrm{U} 2$ that can suppress the temperature-sensitive phenotype of the G53A mutation, we selected cold-resistant revertants. This approach identified eight independent point mutations (Fig. 5A and B) which can be classified as one of three types. The first class of second-site suppressor acts by directly restoring the mismatched base pair in stem IIa by altering C-62 to U, converting the A-C mismatch at the 53-62 base pair to an A-U. This compensatory base change was previously identified by site-directed mutagenesis (2). The second class of suppressor mutation, also initially defined above by site-directed mutagenesis, functions by disrupting pairing between loop IIa and the conserved complementarity. Four point mutations that fall into this class were isolated, and each disrupts one of the two G-C base pairs in the alternative pairing (G-55-C-104 and C-59-G-100). We had already constructed one of these (G100A) by site-directed mutagenesis (Fig. 1A). We predicted that suppression acting through the disruption of the alternative pairing could be accomplished by mutation of loop IIa, and the G55A and C59U suppressors demonstrate this. The third class of suppressors was unexpected and is defined by two point mutations located in the base of the adjacent stem IIb. These two mutations disrupt the G-69-C-84 base pair. Thirty-four of the sixty-five cold-resistant intragenic suppressor strains recovered in this screen were characterized. The frequencies of the independent point mutations are as follows: A53G (true revertants), 9 of 34; G55A, 9 of 34; C59U, 3 of 34; C62U, 1 of 34; G69A, 2 of 34; C84U, 3 of 34; G100A, 1 of 34; and C104U, 6 of 34. Their positions are marked on the structure of the alternatively folded U2 sequence (Fig. 5A). Note that all the intragenic suppressors that we isolated are transitions of the type expected to be induced by ethyl methanesulfonate (8), whereas the true revertant is not.

Strains carrying U2 G53A grow slightly more slowly than the wild type at $36^{\circ} \mathrm{C} \mathrm{(2)} \mathrm{but} \mathrm{are} \mathrm{severely} \mathrm{growth} \mathrm{inhibited} \mathrm{at} 37^{\circ} \mathrm{C}$ (45a). We tested the double mutants carrying G53A and the second-site suppressors for their ability to grow at $37^{\circ} \mathrm{C}$ (Fig.
5B). All suppress the heat-sensitive defect as well, suggesting that the potential to form alternative structures may interfere with the ability of the mutant stem IIa to function at both high and low temperatures.

The RNA folding defects caused by the G53A mutation are partially suppressed by the second-site mutations. To determine the effects of the suppressor mutations on $\mathrm{U} 2$ structure, we probed U2 RNA from the suppressor strains (Fig. 5C). The G53A mutation alone shows an altered pattern of DMS reactivity in comparison with the wild type (49) (Fig. 5C; see also Fig. 3). Decreased reactivity is observed in the nucleotides of loop IIa (A-57, A-58, and C-59), while the accessibility of the stem IIa nucleotides (C-52, A-53, A-61, and C-62) increases. In most cases, the structures of the suppressors show a shift back to the wild-type pattern, with increased reactivities in loop IIa and the conserved complementarity and decreased reactivities in the stem IIa nucleotides, but the distribution and extent of this effect are different for the different suppressors. As shown in Fig. 5C and 3, folding of G53A C62U mutant RNA is more like wild-type folding, leading to protection of A-61 and enhanced reactivity of A-57, A-58, and C-59. The reactivities of C-51 and A-52 (and A-53, which should now be protected by U-62) are only slightly reduced, presumably because the A-53-U-62 base pair is less stable than the wild-type G-53-C-62 base pair and the stem remains subject to disruption. When G53A is combined with either G55A, C59U, $\mathrm{G} 100 \mathrm{~A}$, or $\mathrm{C} 104 \mathrm{U}$ as a double mutant, the reactivities of A-57, A-58, and C-59 are restored, and the protection of the stem IIa nucleotides is slightly improved in comparison with the G53A mutant. The second mutations G69A and C84U weakly improve the accessibility of loop nucleotides A-57, A-58, and C-59 but cause better protection of C-51 and A-52. Even the mismatched A-53 is less reactive in these last two suppressors. An increase in the modification of nucleotides at the base of stem IIb is also seen, caused by the disruption of the G-69C-84 base pair.

The existence of the stem IIb class of suppressors and the structure probing data for the G100A and tmB' mutations in combination with a wild-type stem-loop IIa (Fig. 3 and 5C) 

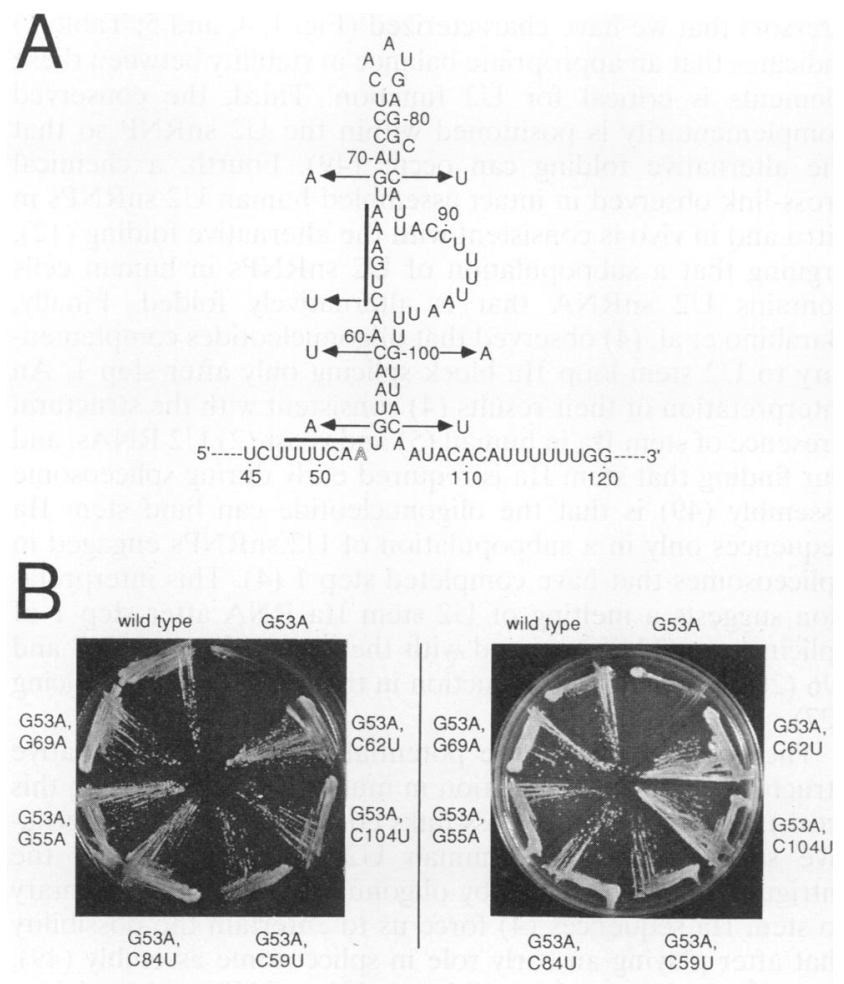

$18^{\circ} \mathrm{C}$
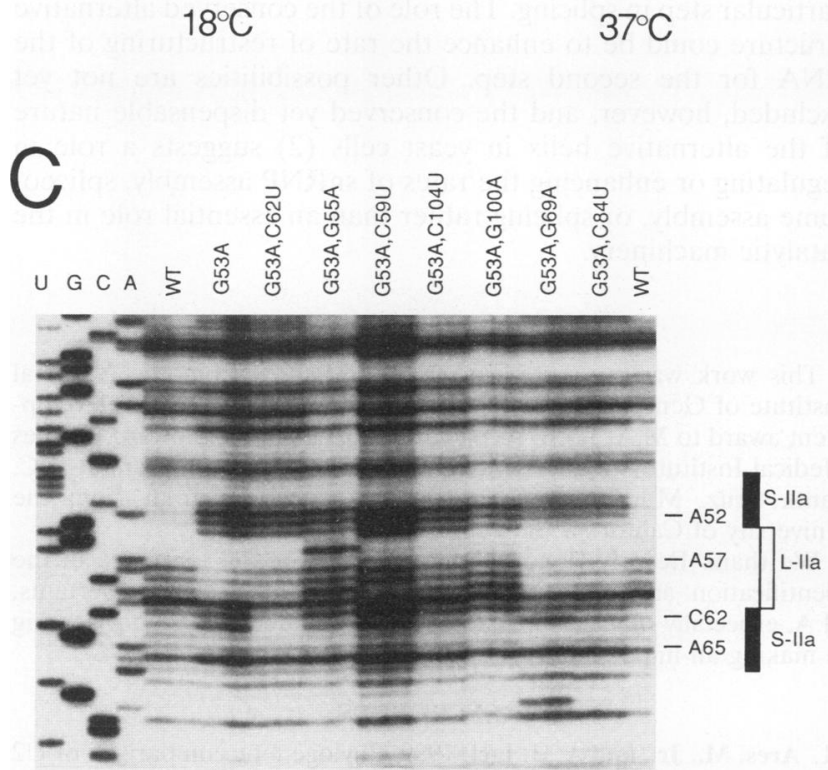

FIG. 5. Intragenic suppressors of U2 G53A. Cold-resistant revertants were generated by ethyl methanesulfonate mutagenesis and analyzed as described in Materials and Methods. (A) Secondary structure model and sequence change in the suppressed U2 snRNAs. The sequence of U2 G53A (2) is shown from nt 45 to 120 in the alternative conformation of Keller and Noon (22). Second-site suppressor mutations are indicated by arrows. (B) Growth phenotypes at 18 and $37^{\circ} \mathrm{C}$ of yeast strains carrying $\mathrm{U} 2$ mutations. An otherwise isogenic set of strains carrying a chromosomal disruption of $U 2$ and the indicated U2 mutant on a centromere plasmid was constructed by plasmid shuffling. Strains were streaked on YEPD and incubated for 2 days at $37^{\circ} \mathrm{C}$ or 4 days at $18^{\circ} \mathrm{C}$. (C) Modification protection analysis of mutant U2 RNAs in vivo. Cultures of a set of otherwise isogenic yeast strains carrying a gene disruption on the chromosome and the indicated U2 allele on a centromere plasmid were grown in liquid YEPD demonstrate the influence of stem IIb on the stability of stem IIa. Note that the two nucleotides following stem IIb are U residues ( $U-86$ and $U-87$ ) and could displace $U-48$ and $U-49$ by pairing with A-66 and A-67 (49). The ability of this isomerization to take place could depend on stacking interactions with the terminal three base pairs of stem IIb. The suppressors destroy this segment of stem IIb and may improve stem IIa stability by blocking the invasion of U-86 and U-87. Consistent with this is the reactivity of A-65 in the wild type. This residue would become reactive as a result of its position at the end of a shortened stem IIa in those molecules in which stem IIb is extended. The conserved complementarity must play a role in allowing this to take place because when it is removed as in the G100A or tmB' mutation, A-65 becomes protected, as does C-62, suggesting a firming up of stem IIa. In addition, C-84 and A-85 at the base of stem IIb adjacent to U-86 and U-87 become reactive. We conclude that the stability of stem IIa is critically regulated by adjacent RNA structures and that the relative stabilities of the alternative structures are delicately balanced. Our mutagenesis and structure probing data, as well as the phylogenetic data, indicate that this balance is important for function and suggest that this region of U2 RNA is dynamic during U2 snRNP assembly or function.

\section{DISCUSSION}

We have demonstrated that the conserved complementarity required for formation of the competing helix is necessary for the cold-sensitive phenotype of the stem IIa mutants. Secondsite mutations that disrupt the base pairing of this region with the loop IIa nucleotides suppress the temperature sensitivity caused by a base mismatch in stem IIa (Fig. 1, 3, and 5). This type of suppression seems specific for mutations in stem IIa (Table 1), although only one cold-sensitive mutation elsewhere in $\mathrm{U} 2$ was available for testing. Biochemical data presented here demonstrate that suppression of cold sensitivity occurs at the level of spliceosome assembly in vitro (Fig. 2). Mutant alleles in which stem IIa is severely disrupted are inviable, but even a weak version of stem IIa can suffice provided the potential for alternative folding is removed by a suppressor mutation (Table 1). Most importantly, the direct assessment of U2 structure provides evidence for the effect of the suppressor mutations on U2 RNA folding, revealing that the suppressor mutations allow more efficient formation of the essential stem-loop IIa structure in spite of the base mismatch in stem Ila due to the primary mutation (Fig. 3 to 5). Taken together, these results indicate that alternative U2 RNA folding is the cause of cold sensitivity in these mutants.

Suppressors act by enhancing correct RNA folding. Alternative RNA folding has been suggested to cause cold sensitivity by temperature-dependent trapping of RNA in a nonfunctional conformation $(10,49)$. In the cold, interconversion between the structures becomes the rate-limiting step, while at permissive temperatures, the correctly folded form is generated with sufficient frequency that the RNA functions. Mutations in U2 that destabilize stem IIa cause cold sensitivity because the alternative helix prevents stem IIa from forming efficiently (49). Suppressors would act by enhancing the melting of the competing helix at low temperatures (10). A

medium at $30^{\circ} \mathrm{C}$ and exposed to DMS, and then the reaction was quenched and RNA was analyzed as in Fig. 2. Positions of migration of the modification-induced stops for the indicated nucleotides are shown at the right. Black bars, stem IIa (S-IIa) sequences; open bars, competing helix sequences. L-IIa, loop IIa; WT, wild type. 
heat-sensitive phenotype (growth at $36^{\circ} \mathrm{C}$ but not at $37^{\circ} \mathrm{C}$ ) is also suppressed by disruption of the alternatively folded structure (Fig. 5). The same mutations that suppress cold sensitivity may act to suppress heat sensitivity by the same mechanism, suppressing alternative folding. In this case, it is necessary to propose that in the mutant at a high temperature, the ratelimiting spliceosome assembly step requiring stem IIa is slowed by the melting of stem IIa. Ability to form the alternative structure would additionally enhance the melting of stem IIa. A suppressor that destabilizes the competing helix may then reduce the rate of stem IIa melting or stabilize stem IIa at high temperatures, resulting in suppression of the heat-sensitive phenotype. (Note that this temperature-sensitive phenotype shifts the maximum growth temperature of the strain down by only 1 to $2^{\circ} \mathrm{C}$ and is not likely due to a severe thermal inactivation of U2 RNA.) At either high or low temperatures, restoring the balance between stem IIa and the competing helix rather than improving the absolute stability of stem IIa suffices to rescue function.

Low-temperature conformational trapping should be more common upon mutation of an RNA than upon mutation of a protein because of the fewer and simpler rules for folding and molecular complementarity among natural nucleic acids. (With only four side chains, leading to two standard base pairs plus the promiscuity of G-U pairing, it is easy to imagine a single nucleotide change in RNA altering the spectrum and stabilities of possible intramolecular interactions.) This situation provides a ready explanation for the large number of cold-sensitive mutations isolated in rRNA $(9,10,33,34,43)$, ribosomal proteins $(13,17,37,44,48)$, snRNA $(2,13 \mathrm{a}, 25,40$, $45)$, and proteins of the splicing apparatus $(21,42)$. Many if not all of these could have at their root the improved stability of an alternatively folded RNA conformer relative to a conformer required for a rate-limiting step. In situations in which a mutant protein leads to or suppresses cold sensitivity $(13,17$, $21,37,40,42,44,48)$, the wild-type protein may be required to stabilize the functional conformer (40) or participate in the catalysis of the interconversion, perhaps as a helicase $(21,35$, 37, 42). According to this view, it is the effect of protein action on the intrinsic dynamics of RNA conformational interconversion that influences function of the RNA, especially in the cold. A prediction of this model is that cold-sensitive mutations will be common when energetically balanced alternative structures are built into RNA, as appears to be the case with U2.

Why conserve two mutually exclusive interconvertible RNA secondary structures? The conformation of U2 containing stem-loop IIa is important for the stable binding of the U2 snRNP to the assembling spliceosome (49). Although the function of the alternative folding formed by pairing of loop IIa to the conserved complementarity remains uncertain, and it is dispensable (2), there are good reasons for thinking that wild-type U2 RNA sometimes adopts this conformation during function. First, the existence and conservation of the sequence complementary to loop IIa is well supported by comparisons of U2 sequences from a diverse group of eukaryotes $(1,18,22)$. We estimated the free-energy changes of formation (14) of the essential stem IIa and the competing helix duplexes by using U2 sequences from different organisms and found that although the primary sequences of these helices vary, stem IIa was more stable by $-0.5 \mathrm{kcal}(1 \mathrm{kcal}=4.184 \mathrm{~kJ}) / \mathrm{mol}$ on average (range of difference between stem IIa and competing helix in individual organisms, +0.3 to $-1.1 \mathrm{kcal} / \mathrm{mol}$ ) than the competing helix. These estimates serve to illustrate that the two mutually exclusive helices are about equivalent in stability and that the balance between them is a conserved feature of U2 snRNA structure. Second, the set of mutations and sup- pressors that we have characterized (Fig. 1, 4, and 5; Table 1) indicates that an appropriate balance in stability between these elements is critical for $\mathrm{U} 2$ function. Third, the conserved complementarity is positioned within the U2 snRNP so that the alternative folding can occur (49). Fourth, a chemical cross-link observed in intact assembled human U2 snRNPs in vitro and in vivo is consistent with the alternative folding (12), arguing that a subpopulation of U2 snRNPs in human cells contains U2 snRNA that is alternatively folded. Finally, Barabino et al. (4) observed that oligonucleotides complementary to U2 stem-loop IIa block splicing only after step 1 . An interpretation of their results (4) consistent with the structural presence of stem IIa in human (5) and yeast (2) U2 RNAs, and our finding that stem IIa is required early during spliceosome assembly (49) is that the oligonucleotide can bind stem IIa sequences only in a subpopulation of U2 snRNPs engaged in spliceosomes that have completed step 1 (4). This interpretation suggests a melting of U2 stem IIa RNA after step 1 of splicing, possibly associated with the events that pair U2 and U6 (26) or promote U2 function in the second step of splicing (27).

The conservation of the potential to form the alternative structure $(1,18)$, its formation in mutant U2 snRNPs $(49$; this work), the internal U2 cross-links consistent with the alternative structure in intact human U2 snRNPs (12) and the intriguing block to step 2 by oligonucleotides complementary to stem IIa sequences (4) force us to entertain the possibility that after playing an early role in spliceosome assembly (49), stem IIa is disrupted in wild-type U2 snRNPs engaged in a particular step in splicing. The role of the conserved alternative structure could be to enhance the rate of restructuring of the RNA for the second step. Other possibilities are not yet excluded, however, and the conserved yet dispensable nature of the alternative helix in yeast cells (2) suggests a role in regulating or enhancing the rates of snRNP assembly, spliceosome assembly, or splicing rather than an essential role in the catalytic machinery.

\section{ACKNOWLEDGMENTS}

This work was supported by grant GM40478 from the National Institute of General Medical Sciences and a research career development award to M.A. J.S.B. received support from the Howard Hughes Medical Institute Summer Undergraduate Research Program at U.C. Santa Cruz. M.I.Z. was supported by a training grant from the University of California Biotechnology Committee.

We thank Beverly Bonde and Andrea Silver for assistance in the identification and characterization of the cold-sensitive revertants. M.A. especially thanks J.S.B. for her mature and professional bearing in making an impossible first authorship decision possible.

\section{REFERENCES}

1. Ares, M., Jr., and A. H. Igel. 1989. Phylogenetic comparison of U2 small nuclear RNA sequences suggests a pseudoknotted structure. UCLA Symp. Mol. Cell. Biol. 94:13-23.

2. Ares, M., Jr., and A. H. Igel. 1990. Lethal and temperature sensitive mutations and their suppressors identify an essential structural element in U2 small nuclear RNA. Genes Dev. 4:21322145.

3. Ausubel, F. M., R. Brent, R. E. Kingston, D. D. Moore, J. G. Seidman, J. A. Smith, and K. Struhl (ed.). 1987. Current protocols in molecular biology, p. 13.3, 13.11.3. Greene Publishers and Associates and Wiley-Interscience, New York.

4. Barabino, S., B. Sproat, and A. Lamond. 1992. Antisense probes targeted to an internal domain in U2 snRNP specifically inhibit the second step of splicing. Nucleic Acids Res. 20:4457-4464.

5. Behrens, S.-E., K. Tyc, B. Kastner, J. Reichelt, and R. Luhrmann. 1993. Small nuclear ribonucleoprotein (RNP) U2 contains numerous additional proteins and has a bipartite RNP structure under 
splicing conditions. Mol. Cell. Biol. 13:307-319.

6. Chattoo, B., F. Sherman, T. Fejellstedt, D. Menhnert, and M. Ogur. 1979. Selection of lys 2 mutants of the yeast Saccharomyces cerevisiae by the utilization of alpha-aminoadipate. Genetics 93:51-65.

7. Cheng, S. C., and J. Abelson. 1987. Spliceosome assembly in yeast. Genes Dev. 1:1014-1027.

8. Coulondre, C., and J. H. Miller. 1977. Genetic studies of the lac repressor. IV. Mutagenic specificity in the lacI gene of Escherichia coli. J. Mol. Biol. 117:577-606.

9. Cui, Z., and T. L. Mason. 1989. A single nucleotide substitution at the rib2 locus of the yeast mitochondrial gene for 21S rRNA confers resistance to erythromycin and cold-sensitive ribosome assembly. Curr. Genet. 16:273-279.

10. Dammel, C., and H. Noller. 1993. A cold-sensitive mutation in 16s rRNA provides evidence for helical switching in ribosome assembly. Genes Dev. 7:660-670.

11. Datta, B., and A. M. Weiner. 1991. Genetic evidence for base pairing between U2 and U6 snRNAs in mammalian mRNA splicing. Nature (London) 352:821-824.

12. Datta, B., and A. M. Weiner. 1992. Cross-linking of U2 snRNA using nitrogen mustard: evidence for higher order structure. J. Biol. Chem. 267:4497-4502.

13. Fearon, K., and T. L. Mason. 1992. Structure and function of MRP20 and MRP49, the nuclear genes for two proteins of the 54S subunit of the yeast mitochondrial ribosome. J. Biol. Chem. 267:5162-5170.

13a.Fortner, D., R. Troy, and D. Brow. 1994. A stem/loop in U6 RNA defines a conformational switch required for pre-mRNA splicing. Genes Dev. 8:221-233.

14. Freier, S., R. Kierzek, J. A. Jaeger, N. Sugimoto, M. H. Caruthers, T. Neilson, and D. H. Turner. 1986. Improved free-energy parameters for predictions of RNA duplex stability. Proc. Natl. Acad. Sci. USA 83:9373-9377.

15. Green, M. R. 1991. Biochemical mechanisms of constitutive and regulated pre-mRNA splicing. Annu. Rev. Cell Biol. 7:559-599.

16. Guthrie, C. 1991. Messenger RNA splicing in yeast: clues to why the spliceosome is a ribonucleoprotein. Science 253:157-163.

17. Guthrie, C., H. Nashimoto, and M. Nomura. 1969. Structure and function of Escherichia coli ribosomes. VII. Cold-sensitive mutations defective in ribosome assembly. Proc. Natl. Acad. Sci. USA 63:384-391.

18. Guthrie, C., and B. Patterson. 1988. Spliceosomal snRNAs. Annu. Rev. Genet. 22:387-419.

19. Igel, A., and M. Ares, Jr. 1988. Internal sequences that distinguish yeast from metazoan $U 2$ are unnecessary for pre-mRNA splicing. Nature (London) 334:450-453.

20. Inoue, T., and T. Cech. 1985. Secondary structure of the circular form of the Tetrahymena rRNA intervening sequence: a technique for RNA structure analysis using chemical probes and reverse transcriptase. Proc. Natl. Acad. Sci. USA 82:648-652.

21. Jamieson, D. J., B. Rahe, J. Pringle, and J. D. Beggs. 1991. A suppressor of a yeast splicing mutation (prp8-1) encodes a putative ATP-dependent RNA helicase. Nature (London) 349:715-717.

22. Keller, E., and W. A. Noon. 1985. Intron splicing: a conserved internal signal in introns of Drosophila pre-mRNAs. Nucleic Acids Res. 13:4971-4981.

23. Kunkel, T. 1985. Rapid and efficient site-specific mutagenesis without phenotypic selection. Proc. Natl. Acad. Sci. USA 82:488492.

24. Lin, R.-J., A. J. Newman, S.-C. Cheng, and J. Abelson. 1985. Yeast mRNA splicing in vitro. J. Biol. Chem. 260:14780-14792.

25. Madhani, H. D., R. Bordonne, and C. Guthrie. 1990. Multiple roles for U6 snRNA in the splicing pathway. Genes Dev. 4:22642277.

26. Madhani, H. D., and C. Guthrie. 1992. A novel base-pairing interaction between $\mathrm{U} 2$ and $\mathrm{U} 6$ snRNAs suggests a mechanism for the catalytic activation of the spliceosome. Cell 71:803-817.

27. McPheeters, D., and J. Abelson. 1992. Mutational analysis of the yeast U2 snRNA suggests a structural similarity to the catalytic core of group I introns. Cell 71:819-831.

28. Milligan, J., and O. Uhlenbeck. 1989. Synthesis of small RNAs using T7 RNA polymerase. Methods Enzymol. 180:51-62.

29. Miraglia, L., S. Seiwert, A. Igel, and M. Ares. 1991. Limited functional equivalence of phylogenetic variation in small nuclear RNA: yeast U2 RNA with altered branchpoint complementarity inhibits splicing and produces a dominant lethal phenotype. Proc. Natl. Acad. Sci. USA 88:7061-7065.

30. Moazed, D., S. Stern, and H. Noller. 1986. Rapid chemical probing of conformation in 16S ribosomal RNA and 30S ribosomal subunits using primer extension. J. Mol. Biol. 187:399-416.

31. Pikielny, C. W., and M. Rosbash. 1986. Specific small nuclear RNAs are associated with yeast spliceosomes. Cell 45:869-877.

32. Pikielny, C. W., B. C. Rymond, and M. Rosbash. 1986. Electrophoresis of ribonucleoproteins reveals an ordered assembly pathway of yeast splicing complexes. Nature (London) 324:341-345.

33. Powers, T., and H. Noller. 1990. Dominant lethal mutations in a conserved loop in 16s rRNA. Proc. Natl. Acad. Sci. USA 87:10421046.

34. Powers, T., and H. Noller. 1991. A functional pseudoknot in 16S ribosomal RNA. EMBO J. 10:2203-2214.

35. Ripmaster, T., G. Vaughn, and J. Woolford. 1992. A putative ATP-dependent RNA helicase involved in Saccharomyces cerevisiae ribosome assembly. Proc. Natl. Acad. Sci. USA 89:1113111135.

36. Ruby, S., and J. Abelson. 1988. An early hierarchic role of U1 small nuclear ribonucleoprotein in spliceosome assembly. Science 242:1028-1035.

37. Sachs, A. B., and R. W. Davis. 1990. Translation initiation and ribosomal biogenesis: involvement of a putative rRNA helicase and RPL46. Science 247:1077-1079.

38. Seraphin, B., and M. Rosbash. 1989. Identification of functional U1 snRNA-pre-mRNA complexes committed to spliceosome assembly and splicing. Cell 59:349-358.

39. Seraphin, B., and M. Rosbash. 1991. The yeast branchpoint sequence is not required for the formation of a stable U1 snRNA-pre-mRNA complex and is recognized in the absence of U2 snRNA. EMBO J. 10:1209-1216.

40. Shannon, K., and C. Guthrie. 1991. Suppressors of a U4 snRNA mutation define a novel U6 snRNP protein with RNA-binding motifs. Genes Dev. 5:773-785.

41. Sherman, F., G. Fink, and J. Hicks. 1986. Methods in yeast genetics. Cold Spring Harbor Laboratory, Cold Spring Harbor, N.Y.

42. Strauss, E., and C. Guthrie. 1991. A cold-sensitive mRNA splicing mutant is a member of the RNA helicase gene family. Genes Dev. 5:629-641.

43. Sweeney, R., C.-H. Yao, and M.-C. Yao. 1990. A mutation in the large subunit ribosomal RNA gene of Tetrahymena confers anisomycin resistance and cold sensitivity. Genetics 127:327-334.

44. Tai, P.-C., D. P. Kessler, and J. Ingraham. 1969. Cold-sensitive mutations in Salmonella typhimurium which affect ribosome synthesis. J. Bacteriol. 97:1298-1304.

45. Tollervey, D., and C. Guthrie. 1985. Deletion of a yeast small nuclear RNA gene impairs growth. EMBO J. 4:3873-3878.

45a.Wang, J., and M. Ares, Jr. Unpublished data.

46. Wassarman, D. A., and J. A. Steitz. 1992. Interactions of small nuclear RNAs with precursor messenger RNA during in vitro splicing. Science 257:1918-1925.

47. Wu, J. A., and H. L. Manley. 1991. Base-pairing between U2 and U6 snRNAs is necessary for splicing of a mammalian pre-mRNA. Nature (London) 352:818-821.

48. Yano, R., and T. Yura. 1989. Suppression of the Escherichia coli $r p o H$ opal mutation by ribosomes lacking S15 protein. J. Bacteriol. 171:1712-1717.

49. Zavanelli, M., and M. Ares, Jr. 1991. Efficient association of U2 snRNPs with pre-mRNA requires an essential U2 RNA structural element. Genes Dev. 5:2521-2533. 